\title{
Validity domain of the Born approximation for seismic waveform modelling in realistic 3-D Earth structure
}

\author{
Laura Parisi, ${ }^{1}$ Ana M. G. Ferreira ${ }^{2,3}$ and Yann Capdeville ${ }^{4}$ \\ ${ }^{1}$ School of Environmental Sciences, University of East Anglia, Norwich, United Kingdom. E-mail: l.parisi@uea.ac.uk \\ ${ }^{2}$ Department of Earth Sciences, University College of London, London, United Kingdom \\ ${ }^{3}$ ICIST, DECivil, Instituto Superior Técnico, Universidade de Lisboa, Portugal \\ ${ }^{4}$ Laboratoire de Planétologie et Géodynamique de Nantes (UMR 6112), CNRS, Université de Nantes, France
}

Accepted 2014 November 11. Received 2014 November 10; in original form 2014 July 31

\begin{abstract}
S U M M A R Y
While the first-order Born approximation is increasingly being used in many seismic tomography efforts, its domain of validity to forward model seismic waveforms has not been quantified in the context of current 3-D earth models yet. We here address this issue by comparing teleseismic synthetic surface waveforms calculated using the Born approximation with spectral element method solutions for a variety of realistic global 3-D earth models. We find that the Born approximation has a very limited domain of validity when applied to seismic waveforms. Specifically, it can only accurately model the phase (amplitude) of surface waveforms for source-receiver paths leading to time shifts smaller than about 15 per cent ( 5 per cent) of the wave period considered. These conditions usually occur in earth models as S20RTS or S40RTS combined with a homogeneous crust for periods longer than $T \sim 80-90 \mathrm{~s}$. For models with stronger heterogeneity and/or realistic 3-D crustal structure, only the phase of waveforms with periods longer than $\sim 120-130 \mathrm{~s}$ can be accurately modelled with the Born approximation.
\end{abstract}

Key words: Surface waves and free oscillations; Seismic tomography; Theoretical seismology; Wave scattering and diffraction; Wave propagation.

\section{INTRODUCTION}

With increasing computational power, current efforts to enhance the quality of global seismic tomographic models involve the inversion of waveforms in order to fully exploit the information contained in seismograms. Despite the recent development of highly accurate forward modelling schemes (e.g. Komatitsch et al. 2002; NissenMeyer et al. 2014) promising a new generation of high-resolution tomographic images, waveform tomography presents some drawbacks, such as the high computational cost and the strong nonlinearity of the inverse problem (e.g. Nolet 2014).

Because of the intensive computing needs of such accurate seismic wavefield calculations, several approximations such as the great circle approximation (Woodhouse \& Dziewonski 1984) and nonlinear asymptotic techniques (Li \& Tanimoto 1993; Li \& Romanowicz 1995) have been developed and used. The Born approximation is a first-order perturbation theory, which is often used in seismology within the normal mode framework (Woodhouse 1980; Tanimoto 1984). It takes into account finite-frequency effects and has been successfully used to calculate phase delay kernels in global tomography applications (e.g. Montelli et al. 2004; Zhou et al. 2006). In this paper, we test the possibility of using the Born approximation as a forward modelling scheme for waveform tomography instead. Its implementation is relatively simple and efficient. Also, it is par- ticularly appealing in seismic tomography because it naturally gives a linear inverse problem. On the other hand, the disadvantage of the Born approximation is that is it a short-time approximation, that is, it is only valid for sufficiently small times, and for weak perturbations compared to the reference model (usually 1-D) (Woodhouse 1980; Tanimoto 1984; Romanowicz et al. 2008). It is thus of primary importance to understand the domain of validity of the Born approximation in relation to the strength of the heterogeneity obtained from tomographic inversions.

In particular, we are here interested in quantifying the domain of validity of the Born approximation when modelling relatively longperiod surface waves in realistic 3-D earth models. Some previous studies have investigated how good the Born approximation is to compute synthetic seismograms and kernels (Capdeville et al. 2002; Tromp et al. 2005; Romanowicz et al. 2008; Panning et al. 2009; Peter et al. 2009; Dalton et al. 2014). However, its accuracy has been examined only by considering a few simplified situations of mantle heterogeneity or for a few existing 3-D tomographic models. Moreover, a general criterion predicting the situations in which it breaks down has not been established yet. We here aim at filling this gap in the framework of forward modelling techniques.

To achieve this goal we calculate time-domain waveforms with the short-time first-order Born approximation (BORN) by using the approach described by Capdeville (2005). This efficient technique 
considers the full mode coupling although not explicitly present in the equations. The effect of the perturbations with respect to the 1-D reference model, which is PREM in this study (Dziewonski \& Anderson 1981), is integrated numerically, leading to compute the sum of the scattered fields induced by a finite number of scattering points. The perturbed wavefield is summed to the 1-D reference seismogram calculated by normal mode summation (NMS, Gilbert 1971). BORN waveforms are then compared with seismograms computed by the spectral element method (SEM, Komatitsch \& Villotte 2002; Komatitsch \& Tromp 2002a,b; Komatitsch et al. 2010), and phase and amplitude accuracy are separately assessed for different dominant wave periods and for a wide range of realistic 3-D earth models.

\section{DATA AND METHOD}

We generate SEM (SPECFEM3D_GLOBE package) and BORN waveforms and systematically compare the corresponding minorarc fundamental mode Rayleigh (R1) and Love (G1) waves. We choose SEM as ground truth because it is a highly accurate purely numerical method for the full calculation of the seismic wavefield in realistic 3-D media and represents a powerful tool to assess the accuracy of approximate forward modelling techniques.

Waveform comparisons are conducted between waveforms filtered around $T \sim 60 \mathrm{~s}, T \sim 100 \mathrm{~s}$ and $T \sim 150 \mathrm{~s}$. Time windows capturing 2.5 cycles of the R1 and G1 arrivals, centred around the maximum amplitude, are manually selected to avoid the interference of surface wave overtones. Errors in phase $\left(E_{\phi}\right)$ and amplitude $\left(E_{\mathrm{A}}\right)$ of time-domain velocity waveforms are estimated for every BORN-SEM pair of waveforms. $E_{\phi}$ is computed as the phase shift in seconds by cross-correlation of the two waveforms. In Figs 3 and $6, E_{\phi}$ is expressed as a percentage of the wave period T. $E_{\mathrm{A}}$ is calculated as:

$E_{A}=100 * \frac{\sum_{i}\left|A_{i+E_{\varphi}}^{B}-A_{i}^{S}\right|}{\sum_{i}\left|A_{i}^{S}\right|}$

where $A^{\mathrm{B}}$ and $A^{\mathrm{S}}$ are the amplitudes of BORN and SEM waveforms, respectively. The summations are carried out over the waveform time samples $i$. The subscript $i+E_{\phi}$ of $A^{\mathrm{B}}$ indicates that the BORN waveform is shifted in time by $E_{\phi}$ seconds before computing the difference $A^{\mathrm{B}}-A^{\mathrm{S}}$. Thus, $E_{\mathrm{A}}$ represents the percentual amplitude difference to the SEM amplitude computed for the best waveform phase-matching. We choose a percentage difference rather than a ratio to indifferently consider the cases in which the BORN amplitudes are either larger or smaller than the SEM ones by the same quantity. We also preferred the absolute value for the difference rather than the squared difference to reduce the influence of outliers. For the same reason, we choose to use the medians of the $E_{\phi}$ and $\mathrm{E}_{\mathrm{A}}$ rather than the average values to summarise the results (presented in Section 3).

To carry out realistic waveform tomography experiments, we calculate theoretical seismograms for three real seismic events ev1, ev2 and ev3 with $\mathrm{Mw}_{\mathrm{ev} 1}=6.4, \mathrm{Mw}_{\mathrm{ev} 2}=6.2$ and $\mathrm{Mw}_{\mathrm{ev} 3}=6.4$ and depth ev1 $=16 \mathrm{~km}$, depth ev2 $=20 \mathrm{~km}$ and depth $\mathrm{ev} 3=15 \mathrm{~km}$. Focal mechanisms are presented in Fig. 1. Earthquakes are selected to sample different regions of the Earth and to excite fundamental mode surface waves well. We select 73 stations from the Global Seismic Network whose epicentral distance from the source ranges between $40^{\circ}$ and $140^{\circ}$. A sketch of the ray coverage is presented in Fig. 1.

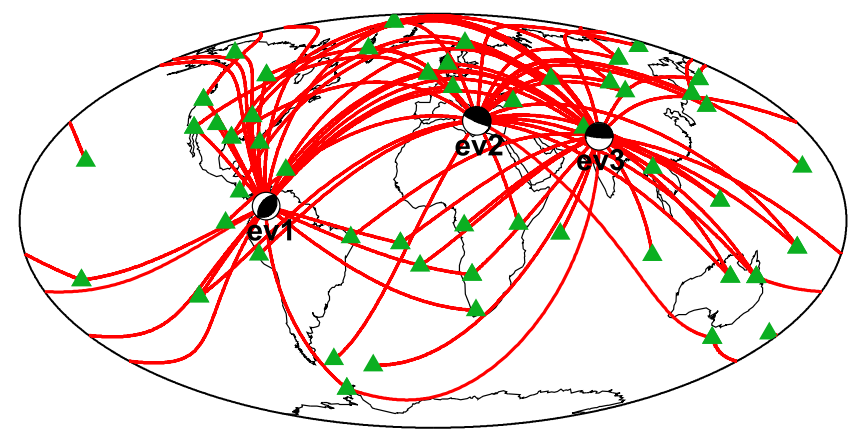

Figure 1. Source-receiver geometry used in this work. The sources ev1, ev2 and ev 3 are at 16, 20 and $15 \mathrm{~km}$ depth and the stations are represented by green triangles.

\subsection{Earth models}

In this study, we adopt six mantle and two crustal models and we combine them to ensure that we use realistic earth models. We start from S20RTS (Ritsema et al. 1999; Figs 2b and g) and S40RTS (Ritsema et al. 2011; Fig. 2d) mantle models. S20RTS and S40RTS are represented by 3-D perturbations in shear wave speed $(\delta V s)$, superimposed upon the spherically symmetric PREM model (Dziewonski \& Anderson 1981). The perturbations in compressional wave speed $\delta V p$ and density $\delta \rho$ are scaled with respect to the $V s$ perturbations, $\delta \rho=0.40 \times \delta V_{S}$ (Anderson et al. 1968) and $\delta V p=0.59 \times \delta V S$ (Robertson \& Woodhouse 1995). We build two other models by multiplying the $\delta V s, \delta V p$ and $\delta \rho$ of S20RTS by the factors $\mathrm{PV}=0.5$ (Fig. 2a) and $\mathrm{PV}=1.75$ (Fig. 2c). $\mathrm{PV}=0.5$ is adopted to build a model with fairly weak heterogeneity compared to current tomographic models. On the other hand, we choose $\mathrm{PV}=1.75$ as end-member model with heterogeneity as strong as in recent high-resolution upper mantle models (e.g. Schaeffer et al. 2013). S40RTS is parameterised with spherical harmonic basis functions expanded up to degree 40 ( $l_{\max }=40$, Fig. $\left.2 d\right)$. We derive two other models from S40RTS by truncating the maximum degree of the spherical harmonics to $20\left(l_{\max }=20\right.$, Fig. $\left.2 \mathrm{e}\right)$ and 12 $\left(l_{\max }=12\right.$, Fig. 2 f), gradually eliminating the smaller scale-lengths of heterogeneity.

At first we couple the six mantle models with a homogeneous crust layer (Moho depth $=24.4 \mathrm{~km}, V s=3.2 \mathrm{~km} \mathrm{~s}^{-1}, V p=$ $5.8 \mathrm{~km} \mathrm{~s}^{-1}, \rho=2.6 \mathrm{~g} \mathrm{~cm}^{-3}$; Figs $2 \mathrm{a}-\mathrm{f}$ ). In addition, we couple S20RTS with a 3-D crustal model derived from CRUST2.0 (Bassin et al. 2000; Fig. $2 \mathrm{~g}$ ). For the sake of simplicity in the calculations and to avoid the need to mesh complex crustal discontinuities in SEM (which could introduce numerical errors), the corresponding crust has a constant Moho depth $(24.4 \mathrm{~km})$. Effects of the increase (or decrease) of the Moho depth with respect to CRUST2.0 are compensated by an increase (or decrease) of the wave speeds above (or below) the Moho, applying the trade-off relationship between the Moho depth and the seismic shear wave speed reported by Lebedev et al. (2013). We carried out extensive tests to ensure that the earth models are consistently implemented in the SEM and BORN codes.

\section{RESULTS}

We calculate $E_{\phi}$ and $\mathrm{E}_{\mathrm{A}}$ for BORN-SEM pairs of R1 and $\mathrm{G} 1$ waveforms at $T \sim 60,100$ and $150 \mathrm{~s}$ for every path shown in Fig. 1. For each model, the medians of $E_{\Phi}$, expressed as percentage of a wave cycle, and $E_{\mathrm{A}}$, as defined by eq. (1), are summarized in Fig. 3. The summary (Fig. 3) highlights that: (i) the fit between BORN and SEM calculations deteriorates both in amplitude and phase as 


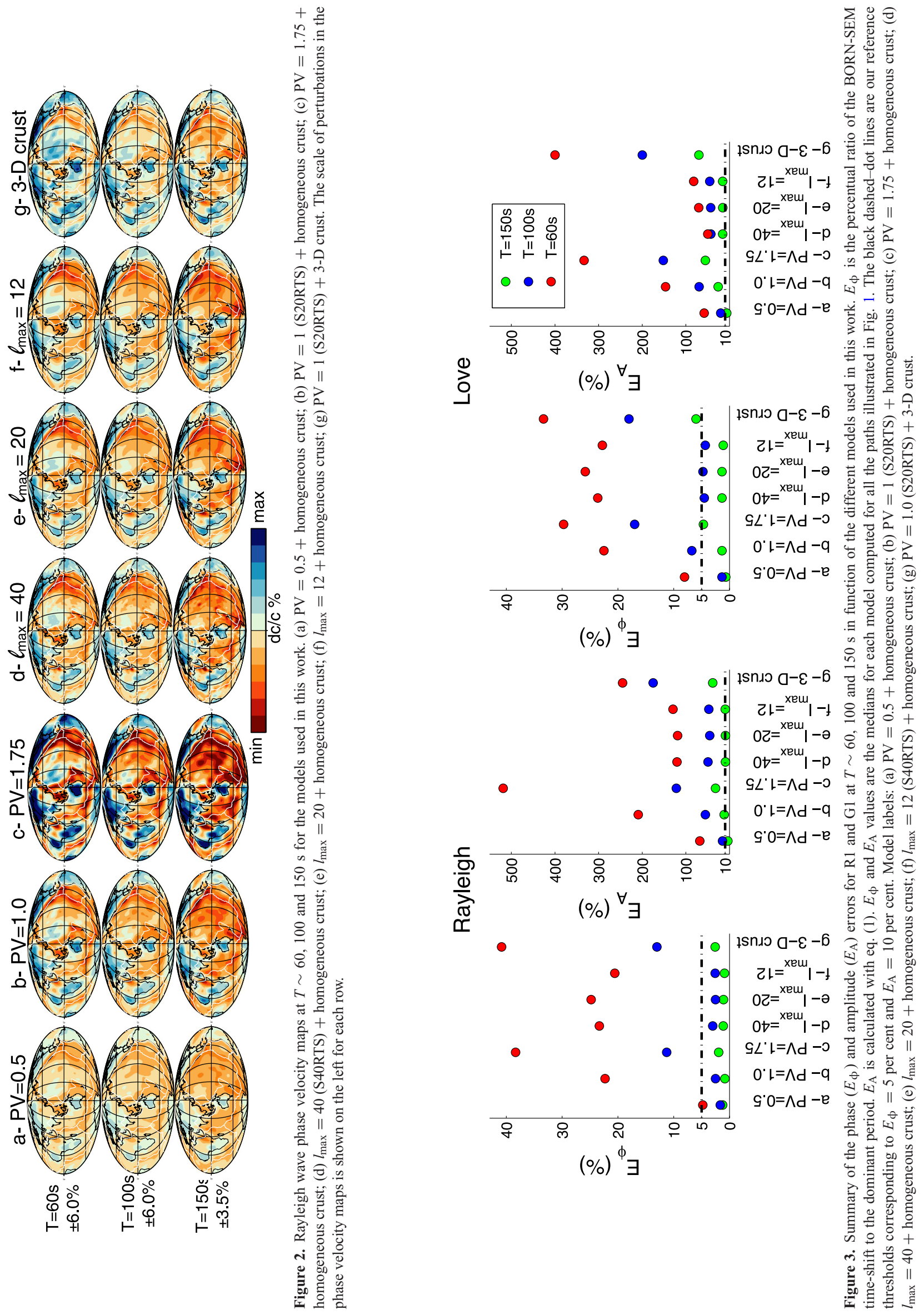


the dominant period decreases and is particularly poor at $T \sim 60 \mathrm{~s}$; (ii) a worsening of the fit is also associated with the increase of the PV factor; (iii) using a 3-D crustal model rather than the homogeneous layer yields a poorer BORN-SEM match and (iv) BORN is not affected by the scale length of heterogeneity of the model (expressed by $l_{\max }$ ). These considerations are valid regardless of the type of waves (Rayleigh or Love).

By visual inspection of the waveforms and associated errors, we setup error thresholds to define a good modelling. These thresholds are 5 per cent of a wave cycle for $E_{\phi}$ and 10 per cent for $\mathrm{E}_{\mathrm{A}}$. For illustration, examples of waveform comparisons for various values of $E_{\phi}$ and $E_{\mathrm{A}}$ are shown in Fig. 4. By means of these thresholds (black dashed-dot lines in Fig. 3), it arises that BORN matches well the phase of Rayleigh waves predicted by SEM only in case of mantle models with: (i) $\mathrm{PV}=0.5$ for $T>60 \mathrm{~s}$; (ii) $\mathrm{PV}=1.0$, such as S20RTS (but also S40RTS and models derived from it) for $\mathrm{T}>100 \mathrm{~s}$; (iii) $\mathrm{PV}=1.75$ and $\mathrm{PV}=1.0$ mantle when the latter is combined with a heterogeneous crust model similar to CRUST2.0, for $T>150$. On the other hand, surface wave amplitudes modelled with BORN only match SEM results for waves with $T \sim 150 \mathrm{~s}$ and for the models: (i) S20RTS with PV $=0.5$; (ii) S40RTS and (iii) models derived from S40RTS with $l_{\max }=20$ and 12. Regarding Love waves, the performance of the Born approximation follows similar trends but with slightly poorer fits to SEM results. On the
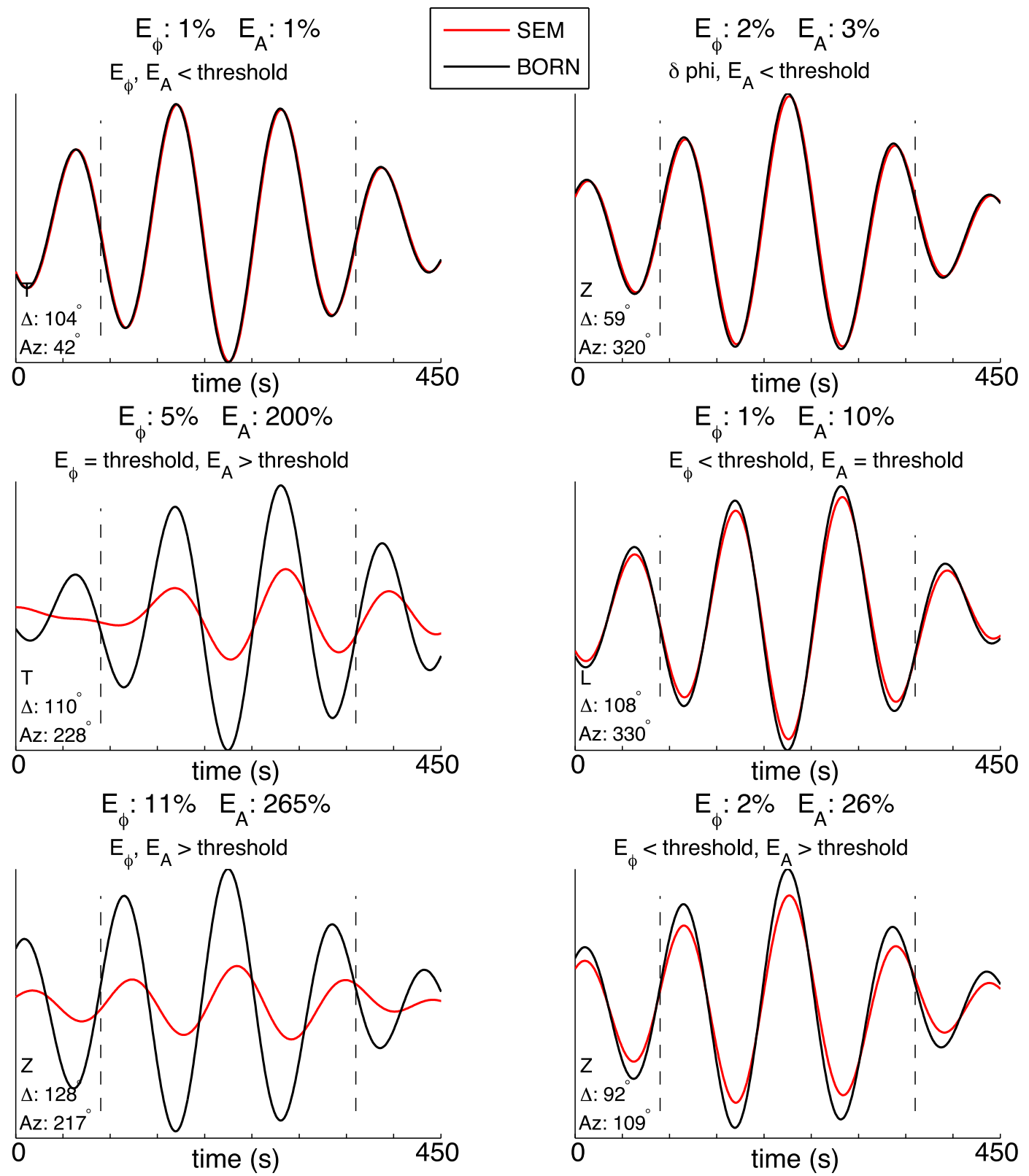

Figure 4. BORN-SEM comparison examples of R1 ( $Z$ and $L$ components) and $\mathrm{G} 1$ ( $T$ component) waveforms at $T \sim 100 \mathrm{~s}$ for the event ev1. $E_{\phi}$ and $E_{\mathrm{A}}$ are reported above each pair specifying the relation with the threshold of goodness used in this work ( $E_{\phi}=5$ per cent and $E_{\mathrm{A}}=10$ per cent). Seismogram component, epicentral distance and azimuth are indicated in the left-bottom corner. The black dashed lines indicate the selected time windows for the errors calculations. 

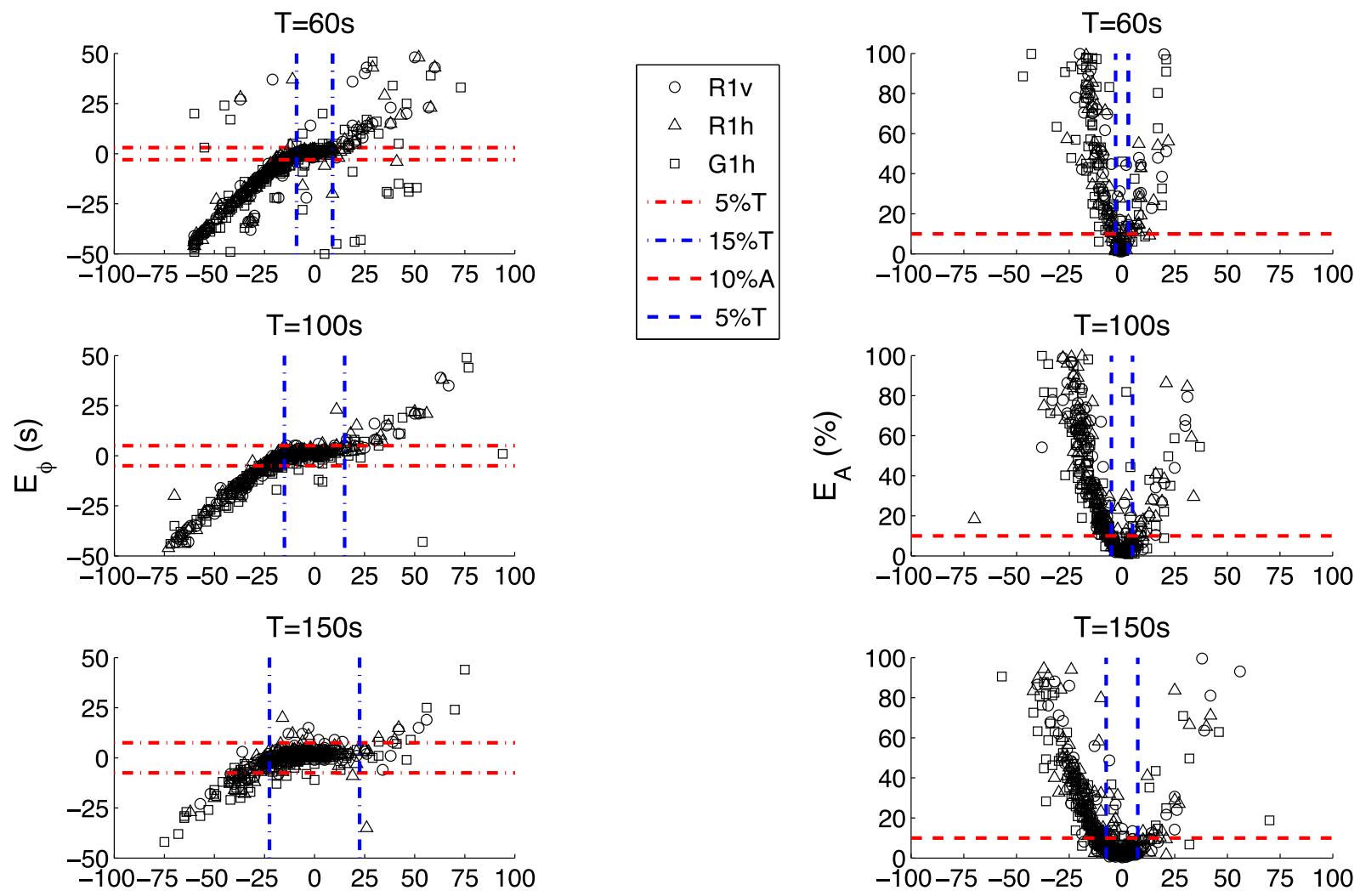

$\delta \phi(\mathrm{s})$

$\delta \phi(\mathrm{s})$

Figure 5. Phase $\left(E_{\phi}\right)$ and amplitude $\left(E_{\mathrm{A}}\right)$ errors of BORN with respect to SEM synthetics at $T \sim 60,100$ and $150 \mathrm{~s}$, for PV $=0.5,1.0$ (S20RTS) and 1.75 mantle models combined with a homogeneous crustal layer versus the accumulated phase-delay on the path $(\delta \Phi)$. Each point corresponds to a BORN-SEM pair. $\delta \Phi$ is the time-shift between SEM3-D and SEM1-D waveforms. $E_{\phi}$ is calculated as time-shift between a BORN-SEM pair and $E_{\mathrm{A}}$ using eq. (1) for each BORN-SEM pair. Only $-50 \mathrm{~s}<E_{\phi}<50 \mathrm{~s}$ and $E_{\mathrm{A}}<100$ per cent are included to zoom the plot around the window of validity. R1v and R1h refer to Rayleigh waves windowed in the vertical and radial components of the seismogram, respectively. G1h refers to Love waves windowed in the transverse component. Red lines are the reference thresholds $\left(E_{\phi}= \pm 5\right.$ per cent and $E_{\mathrm{A}}=10$ per cent). Blue lines enclose the BORN validity domain, $\delta \Phi<15$ per cent for the phase and $\delta \Phi<5$ per cent for the amplitude. See Section 3.1 for more details.

whole, the performance of the Born approximation is not encouraging in the framework of the forward modelling for waveform tomography based on current 3-D tomographic models.

\subsection{Domain of validity}

In order to define the domain of validity of BORN applied to waveforms, we analyse the correlation between the individual BORN errors and the phase delays in seconds accumulated along the paths $(\delta \Phi)$, with respect to the reference waveforms (Fig. 5). Namely, $\delta \Phi$ is the phase-shift in seconds between the SEM seismogram for a 3-D earth model and the NMS reference waveform calculated for PREM. $\delta \Phi$ is calculated by cross-correlation and it is expected to be linearly related to the perturbations of the 3-D model along the path (Mercerat \& Nolet 2013). Fig. 5 shows that BORN fits well SEM in phase when $\delta \Phi$ is less than 15 per cent of a waveform cycle, quantifying a narrow but well-defined domain of validity. For $\delta \Phi$ larger than 15 per cent, $E_{\phi}$ linearly grows with $\delta \Phi$. This behaviour is valid for all the periods and models considered. A correlation between $E_{\mathrm{A}}$ and $\delta \Phi$ is also reported in Fig. 5. The validity domain for the amplitude modelling is narrower $(\delta \Phi<5$ per cent $)$ and less defined. Note that $E_{\phi}$ and $E_{\mathrm{A}}$ against $\delta \Phi$ show a similar behaviour for both Rayleigh and Love waves (represented in Fig. 5 with different symbols).

\section{DISCUSSION}

The Born approximation is a short-time approximation, which depends on: (i) the strength and scale of heterogeneity; (ii) the number of wave cycles in the heterogeneous regions, which in turn depends on the wave frequency and path length. Indeed, increasing the wave frequency will lower the wavelength and thus increase the number of wave cycles in a given fixed heterogeneous region and fixed time window length. Likewise, using longer paths and thus longer time windows to reach seismic surface waves leads to a larger number of wave cycles. To study the effects of the short-time condition, we analyse $E_{\phi}$ as a function of the epicentral distance (Fig. 6). As a low correlation between the errors and distance is found for all the periods considered, we only show the correlation of $E_{\phi}$ for $T \sim$ $100 \mathrm{~s}$ for conciseness. Despite the average error (green line, Fig. 6) showing a slightly positive slope, the large amount of small errors at large distances suggests that the Born approximation can virtually model well surface waves propagating even at distances of $140^{\circ}$ (maximum distance considered in this work). This is due to the fact that the heterogeneity in 3-D earth models is not distributed uniformly. Thus, the performance of the Born approximation is more strongly correlated with the total phase delay accumulated along the path $(\delta \Phi)$ than with the path length.

This fact explains why the medians of the errors rise with strength of heterogeneity of the 3-D earth models (expressed by the PV 


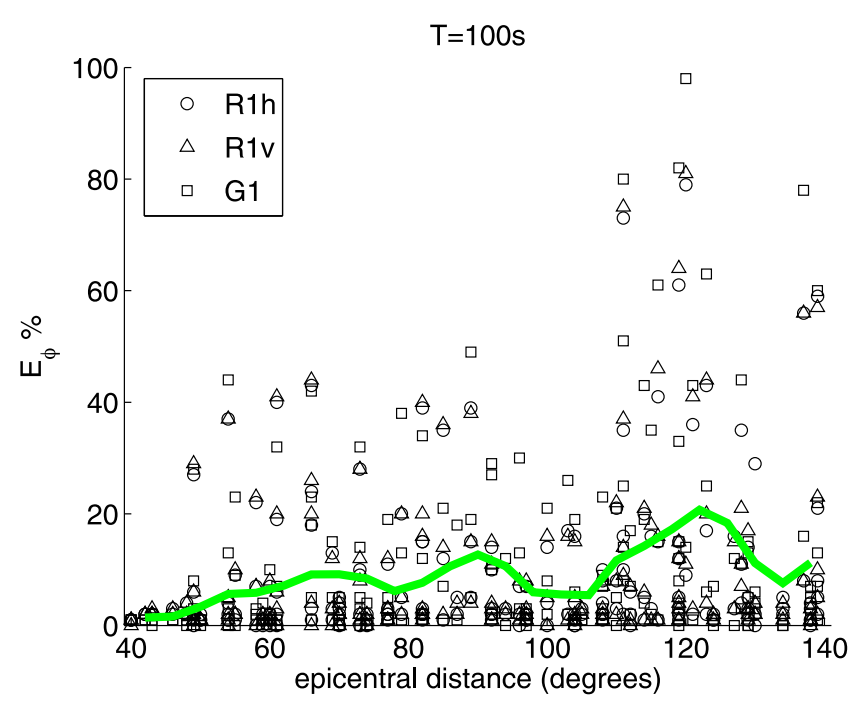

Figure 6. Correlation between $E_{\phi}$ (in percentage) and the epicentral distance at $T \sim 100 \mathrm{~s}$ for $\mathrm{PV}=0.5,1.0$ (S20RTS) and 1.75 mantle models combined with a homogeneous crustal layer. R1v and R1h refer to Rayleigh waves windowed in the vertical and radial components of seismogram, respectively. G1h refers to Love waves windowed in the transverse component. In green, a moving average with a window of $4^{\circ}$ is shown.

factor) and the frequency of the wave. In fact, because of the dispersion of surface waves, the shorter is the period the shallower are the regions of the Earth sampled by the wave. Conversely, for longer period surface waves the sampled regions include larger depths with weaker heterogeneity. This can be easily noticed from the phase velocity maps at different periods presented in Fig. 2. Thus, we can expect that at periods shorter than $60 \mathrm{~s}$ BORN will perform even worse due to the even stronger heterogeneity in the uppermost parts of the mantle. Also, the slightly larger discrepancies obtained for Love waves (Fig. 3) are indeed related to the stronger heterogeneities in the Love phase velocity maps. In fact, in our calculations, the Love wave phase velocity maps (not shown for brevity) have on average anomalies 20 per cent stronger than the corresponding Rayleigh maps. Finally, the observed worsening in the performance of BORN when using a 3-D crust compared to a homogeneous crustal model is due to the large heterogeneities in CRUST2.0

The high correlation between BORN's performance and $\delta \Phi$ is confirmed by the domain of validity shown in Fig. 5. Our analysis shows that the Born approximation can accurately model the waveform phase of Rayleigh and Love waves for $\delta \Phi$ not exceeding 15 per cent of the wave period and the waveform amplitude for $\delta \Phi$ not exceeding 5 per cent of the period. This condition is more stringent than the rough estimate of 25 per cent reported by Panning et al. (2009). Our findings are in agreement with those presented by Dalton et al. (2014), which used the technique of Zhou et al. (2004) to calculate amplitude kernels based on the Born approximation. The authors showed that the misfits decrease with the dominant period and for $T>75 \mathrm{~s}$ the misfits also decrease with the epicentral distance.

\section{CONCLUSIONS}

Given the increasing interest in forward modelling schemes for seismic tomography taking finite frequency effects into account, the Born approximation is highly appealing due to its simplicity and relative efficiency. However, this method is valid in a limited range of seismic velocity perturbations and time windows, which prompted us to quantify its domain of validity for forward modelling surface waveforms in a range of realistic current 3-D earth models. Previous studies (e.g. Capdeville et al. 2002; Romanowicz et al. 2008; Panning et al. 2009; Peter et al. 2009; Dalton et al. 2014) investigated the accuracy of the Born approximation but a unique criterion defining its domain of validity has not been determined yet, especially regarding the forward modelling of seismic waveforms. We thus generated synthetic seismograms by using the Born approximation for seven different realistic combinations of 3-D mantle and crustal models and we treat SEM synthetics as ground truth. We separately considered phase and amplitude errors between BORN and SEM synthetics at $T \sim 60,100$ and $150 \mathrm{~s}$. We found that the Born approximation can only accurately model the phase of surface waveforms for source-receiver paths leading to time shifts smaller than about 15 per cent of the wave period considered. Regarding the amplitude modelling, the time-shifts cannot exceed 5 per cent of the period. In practice, these conditions are met for 3-D earth structure with the same power of heterogeneity as in the S20RTS or S40RTS models, or lower, at wave periods longer than $80-90 \mathrm{~s}$ for the phase and $150 \mathrm{~s}$ for the amplitude. Hence, the short-time first-order Born approximation has a very restricted domain of validity when applied directly to waveforms. More accurate forward modelling schemes should be used in waveform tomography, or, alternatively, inversions based on secondary measurements such as phase delays or amplitude anomalies remain an attractive possibility.

\section{ACKNOWLEDGEMENTS}

We thank editor Michael Ritzwoller and reviewers Lapo Boschi, Guust Nolet for their detailed and encouraging reviews, which helped improve our manuscript. This research was carried out on the High Performance Computing Cluster supported by the Research and Specialist Computing Support services at the University of East Anglia and on HECToR, the UK National Supercomputing Service. Some of the computations were also performed on the Centre de Calcul Intensif des Pays de la Loire (CCIPL) computers. Some figures were realized using Generic Mapping Tools (GMT; Wessel and Smith 1998). This work was supported by the European Commission's Initial Training Network project QUEST (contract FP7-PEOPLE-ITN-2008-238007, http://www.quest-itn.org). AMGF also thanks funding by the Fundacao para a Ciencia e Tecnologia (FCT) project AQUAREL (PTDC/CTE-GIX/116819/2010).

\section{REFERENCES}

Anderson, D.L., Schreiber, E., Liebermann, R.C. \& Soga, N., 1968. Some elastic constant data on minerals relevant to geophysics, Rev. Geophys., 6, 491-524.

Bassin, C., Laske, G. \& Masters, G., 2000. The current limits of resolution for surface wave tomography in North America, EOS, Trans. Am. geophys. Un. F897, 81.

Capdeville, Y., 2005. An efficient Born normal mode method to compute sensitivity kernels and synthetic seismograms in the earth, Geophys. J. Int., 158, 141-168.

Capdeville, Y., Larmat, C., Vilotte, J.P. \& Montagner, J.P., 2002. Numerical simulation of the scattering induced by a localized plume-like anomaly using a coupled spectral element and modal solution method, Geophys. Res. Lett., 29, 9, doi:10.1029/2001GL013747. 
Dalton, C.A., Hjorleifsdottir, V. \& Ekstrom, G., 2014. A comparison of approaches to the prediction of surface wave amplitude, Geophys. J. Int., 196, 386-404.

Dziewonski, A. \& Anderson, D., 1981. Preliminary reference earth model, Phys. Earth planet. Inter., 25, 297-356.

Gilbert, F., 1971. Excitation of normal modes of the Earth by earthquake sources, Geophys. J. R. astr. Soc., 22, 326-333.

Komatitsch, D. \& Tromp, J., 2002a. Spectral-element simulations of global seismic wave propagation - I. Validation, Geophys. J. Int., 149, 390-412.

Komatitsch, D. \& Tromp, J., 2002b. Spectral-element simulations of global seismic wave propagation - II. Three-dimensional models, oceans, rotation and self-gravitation, Geophys. J. Int., 150, 308-318.

Komatitsch, D. \& Villotte, J.P., 1998. The spectral element method: an efficient tool to simulate the seismic response of 2D and 3D geological structures, Bull. seism. Soc. Am., 88, 368-392.

Komatitsch, D., Ritsema, J. \& Tromp, J., 2002. The spectral-element method, beowulf computing, and global seismology, Science, 298, 1737-1742.

Komatitsch, D., Erlebacher, G., Gddeke, D. \& Michea, D., 2010. High-order finite-element seismic wave propagation modeling with MPI on a large GPU cluster, J. Comput. Phys., 229, 7692-7714.

Lebedev, S., Adam, J.M.-C. \& Meier, T., 2013. Mapping the Moho with seismic surface waves: a review, resolution analysis, and recommended inversion strategies, Tectonophysics, 609, 377-394.

Li, X.-D. \& Romanowicz, B., 1995. Comparison of global waveform inversions with and without considering cross-branch modal coupling, Geophys. J. Int., 121, 695-709.

Li, X.-D. \& Tanimoto, T., 1993. Waveforms of long-period body waves in a slightly aspherical earth model, Geophys. J. Int., 112, 92-102.

Montelli, R., Nolet, G., Dahlen, F.A., Masters, G., Engdahl, E.R. \& Hung, S.-H., 2004. Finite-frequency tomography reveals a variety of plumes in the mantle, Science, 303, 338-343.

Mercerat, D.E. \& Nolet, G., 2013. On the linearity of cross-correlation delay times in finite-frequency tomography, Geophys. J. Int., 192, 681-687.

Nissen-Meyer, T., van Driel, M., Stahler, S.C., Hosseini, K., Hempel, S., Auer, L., Colombi, A. \& Fournier, A. 2014. AxiSEM: broadband 3-D seismic wavefields in axisymmetric media, Solid Earth, 5, 425445 .

Nolet, G., 2014. Transmission tomography in seismology, in Handbook of Geomathematics, eds Freeden, W., Nashed, Z. \& Sonar, Th., Springer, doi:10.1007/978-3-642-27793-1_58-1.
Panning, M.P., Capdeville, Y. \& Romanowicz, B., 2009. Seismic waveform modelling in a 3-D Earth using the Born approximation: potential shortcomings and a remedy, Geophys. J. Int., 177, 161-178.

Peter, D., Boschi, L. \& Woodhouse, J.H., 2009. Tomographic resolution of ray and finite-frequency methods: a membrane-wave investigation, Geophys. J. Int., 177, 624-638.

Ritsema, J., van Heijst, H. \& Woodhouse, J., 1999. Complex shear wave velocity structure imaged beneath Africa and Iceland, Science, 286, 19251928.

Ritsema, J., van Heijst, H., Deuss, A. \& Woodhouse, J.H., 2011. S40RTS: a degree-40 shear-velocity model for the mantle from new Rayleigh wave dispersion, teleseismic traveltime and normal-mode splitting function measurements, Geophys. J. Int., 184, 1223-1236.

Robertson, G. \& Woodhouse, J., 1995. Evidence for proportionality of P and $\mathrm{S}$ heterogeneity in the lower mantle, Geophys. J. Int., 123, 85-116.

Romanowicz, B., Panning, M., Gung, Y. \& Capdeville, Y., 2008. On the computation of long period seismograms in a 3D Earth using normal mode based approximations, Geophys. J. Int., 175, 520-536.

Schaeffer, A.J. \& Lebedev, S., 2013. Global shear speed structure of the upper mantle and transition zone approximations, Geophys. J. Int., 194, 417-449.

Tanimoto, T., 1984. A simple derivation of the formula to calculate synthetic long-period seismograms in a heterogeneous Earth by normal mode summation, Geophys. J. R. astr. Soc., 77, 275-278.

Tromp, J., Tape, C. \& Lin, Q., 2005. Seismic tomography, adjoint methods, time-reversal and banana-doughnut kernels, Geophys. J. Int., 160, 195-216.

Wessel, P. \& Smith, W.H.F., 1998. New improved version of the Generic Mapping Tools released, EOS, Trans. Am. geophys. Un., 79(47), 579, doi:10.1029/98EO00426.

Woodhouse, J., 1980. The coupling and attenuation of nearly resonant multiplets in the Earths free oscillation spectrum, Geophys. J. R. astr. Soc., 61, 261-283.

Woodhouse, J. \& Dziewonski, A., 1984. Mapping the upper mantle: three dimensional modeling of Earth structure by inversion of seismic waveforms, J. geophys. Res., 89, 5953-5986.

Zhou, Y., Dahlen, F. \& Nolet, G., 2004. Three-dimensional Frechet differential kernels for seismic delay times, Geophys. J. Int., 158, 141-168.

Zhou, Y., Nolet, G., Dahlen, F.A. \& Laske, G., 2006. Global upper-mantle structure from finite-frequency surface-wave tomography, J. geophys. Res., 111, doi:10.1029/2005JB003677. 